\title{
String Inspired Singlet Extensions of the Minimal Supersymmetric Standard Model
}

\author{
Tianjun $\mathrm{Li}^{1,2}$ \\ ${ }^{1}$ George P. and Cynthia W. Mitchell Institute for Fundamental Physics, \\ Texas A\&M University, College Station, TX 77843, USA \\ ${ }^{2}$ Institute of Theoretical Physics, Chinese Academy of Sciences, Beijing 100080, China
}

\begin{abstract}
The only allowed Higgs superpotential term at stringy tree level in the string derived Singlet Extensions of the Minimal Supersymmetric Standard Model (SEMSSM) is $h S H_{d} H_{u}$, which leads to an additional global $U(1)$ symmetry in the Higgs potential. We propose the string inspired SEMSSM where the global $U(1)$ symmetry is broken by the additional superpotential terms or supersymmetry breaking soft terms that can be obtained naturally due to the instanton effects or anomalous $U(1)_{A}$ gauge symmetry. In these models, we can solve the $\mu$ problem and the fine-tuning problem for the lightest CP-even Higgs boson mass in the MSSM, generate the baryon asymmetry via electroweak baryogenesis, and predict the new Higgs physics which can be tested at the LHC and ILC.
\end{abstract}

PACS numbers: $11.25 . \mathrm{Mj}, 12.10 . \mathrm{Kt}, 12.60 . \mathrm{Fr}$

Introduction - The Minimal Supersymmetric Standard Model (MSSM) can solve the gauge hierarchy problem elegantly due to supersymmetry, has neutralino as cold dark matter candidate, and accommodates the gauge coupling unification [1, 2]. So, it is the most natural extension of the Standard Model (SM). However, there are a few problems within the MSSM. The bilinear supersymmetric Higgs mass term $\mu H_{d} H_{u}$ in the superpotential, where $H_{d}$ and $H_{u}$ are one pair of Higgs doublets, does not violate supersymmetry and gauge symmetry. Then the natural scale for $\mu$ is about Planck scale but not the $\mathrm{TeV}$ scale, which leads to the $\mu$ problem. Moreover, in order to have the lightest CP-even Higgs boson mass larger than the low bound $114 \mathrm{GeV}$ from the LEP experiment, there exists a few percent fine-tuning [3].

To solve the $\mu$ problem, the Next to the Minimal Supersymmetric Standard Model (NMSSM) was proposed in which a SM singlet $S$ and a $Z_{3}$ discrete symmetry are introduced [4]. The $\mu H_{d} H_{u}$ term is forbidden by the $Z_{3}$ symmetry, and the superpotential in the NMSSM is

$$
W=h S H_{d} H_{u}+\frac{\kappa}{3 !} S^{3},
$$

where $h$ and $\kappa$ are Yukawa couplings. After $S$ obtains a vacuum expectation value (VEV), the effective $\mu$ term $\mu_{\mathrm{eff}}=h\langle S\rangle$ is generated. Also, the F-term of $S$ will give additional contribution to the Higgs quartic coupling, and then can increase the lightest CP-even Higgs boson mass. In addition, the lightest CP-even Higgs boson in the NMSSM can have mass around $100 \mathrm{GeV}$ because of its invisible decay [5], and the above fine-tuning problem for its mass can be solved. Moreover, the observed baryon asymmetry can be generated via electroweak baryogenesis because there are extra $\mathrm{CP}$ violating phases in the supersymmetry breaking soft parameters and the trilinear soft term $A_{h} h S H_{d} H_{u}$ can give us strong first order electroweak phase transition [6]. Similar results hold for the nearly MSSM [7] and the $U(1)^{\prime}$-extended supersymmetric Standard Models [8]. Therefore, the Singlet Extensions of the Minimal Supersymmetric Standard Model
(SEMSSM) is very interesting from phenomenological point of view.

On the other hand, string theory may be the only known theory which can correctly describe the quantum gravity. In string models, we may solve the $\mu$ problem in the MSSM [9], and the doublet-triplet splitting problem in the Grand Unified Theories (GUTs) 10, 11]. Thus, how to indirectly test string models at the Large Hadron Collider (LHC) and the International Linear Collider (ILC) is a pretty interesting question.

As we know, in the string model building, the renormalizable terms in the superpotential, which arise from the Chern-Simmons terms in the heterotic string compactification [12] or instanton effects (triangles formed by the intersections of D6-branes) in Type IIA intersecting D6-brane models [13], have the following generic trilinear form at stringy tree level

$$
W=y_{\phi} \phi_{1} \phi_{2} \phi_{3}
$$

where $y_{\phi}$ is the Yukawa coupling, and $\phi_{i}$ are different fields. Thus, the $\mu H_{d} H_{u}$ term in the MSSM and the $\kappa S^{3} / 3$ ! term in the NMSSM do not exist at stringy tree level in the string derived models. And only the first term $h S H_{d} H_{u}$ in the superpotential in Eq. (11) is allowed where $S$ is a modulus. With only $h S H_{d} H_{u}$ term in the superpotential, we have two global $U(1)$ symmetries in the Higgs potential in which one of them is $U(1)_{Y}$ gauge symmetry. So, there is one global $U(1)$ symmetry, and then there exists one massless Goldstone boson with $S$, $H_{d}^{0}$ and $H_{u}^{0}$ mixing components, which is excluded from the known experiments.

In this letter, we propose the string inspired SEMSSM. The global $U(1)$ symmetry in the Higgs potential is broken by the additional superpotential terms or supersymmetry breaking soft terms. The extra superpotential terms can be realized in the string derived models via instanton effects [9]. With anomalous $U(1)_{A}$ gauge symmetry [14], we construct four simple and concrete SEMSSM. In these models, we can naturally solve the $\mu$ problem and 
the fine-tuning problem for the lightest CP-even Higgs boson mass in the MSSM. We also calculate the Higgs boson masses, chargino masses and neutralino masses at tree level, and predict the new Higgs physics which can be tested at the LHC and ILC. A more detail discussions will be presented elsewhere [15].

Model Building - Let us consider the most general SEMSSM. The generic superpotential is

$$
W=h S H_{d} H_{u}+\mu H_{d} H_{u}+m^{2} S+\frac{\mu^{\prime}}{2 !} S^{2}+\frac{\kappa}{3 !} S^{3},
$$

where $\mu, m^{2}$, and $\mu^{\prime}$ are mass parameters. The corresponding $F$-term scalar potential is

$$
\begin{aligned}
V_{F}= & \left|h H_{d} H_{u}+m^{2}+\mu^{\prime} S+\frac{\kappa}{2 !} S^{2}\right|^{2} \\
& +|h S+\mu|^{2}\left|H_{u}\right|^{2}+|h S+\mu|^{2}\left|H_{d}\right|^{2} .
\end{aligned}
$$

And the $D$-term scalar potential is

$$
V_{D}=\frac{G^{2}}{8}\left(\left|H_{u}\right|^{2}-\left|H_{d}\right|^{2}\right)^{2},
$$

where $G^{2}=g_{Y}^{2}+g_{2}^{2} ; g_{Y}$ and $g_{2}$ are respectively the coupling constants for $U(1)_{Y}$ and $S U(2)_{L}$. Moreover, we introduce the supersymmetry breaking soft terms $V_{\text {soft }}^{I}$ and $V_{\text {soft }}^{I I}$ as follows

$$
\begin{aligned}
V_{\text {soft }}^{I}= & m_{H_{d}}^{2}\left|H_{d}\right|^{2}+m_{H_{u}}^{2}\left|H_{u}\right|^{2}+m_{S}^{2}|S|^{2}, \\
V_{\text {soft }}^{I I}= & -\left(A_{h} h S H_{d} H_{u}+B \mu_{B} H_{d} H_{u}+A_{X} m_{X}^{2} S\right. \\
& \left.+\frac{1}{2 !} B^{\prime} \mu_{B}^{\prime} S^{2}+\frac{1}{3 !} A_{\kappa} \kappa_{X} S^{3}+\text { H.C. }\right)
\end{aligned}
$$

where $m_{H_{d}}^{2}, m_{H_{u}}^{2}$, and $m_{S}^{2}$ are supersymmetry breaking soft masses, $A_{h}, B, \mu_{B}, A_{X}, m_{X}^{2}, B^{\prime}, \mu_{B}^{\prime}$, and $A_{\kappa}$ are supersymmetry breaking soft mass parameters, and $\kappa_{X}$ is the coupling constant. In addition, if $\mu \neq 0, m^{2} \neq 0$, $\mu^{\prime} \neq 0$, or $\kappa \neq 0$, we assume $\mu_{B}=\mu, m_{X}^{2}=m^{2}, \mu_{B}^{\prime}=$ $\mu^{\prime}$, or $\kappa_{X}=\kappa$, respectively. However, even if $\mu=0$, $m^{2}=0, \mu^{\prime}=0$, or $\kappa=0$, we can show that $\mu_{B}, m_{X}^{2}, \mu_{B}^{\prime}$, or $\kappa_{X}$ might not be zero in general, so the global $U(1)$ symmetry in the Higgs potential can be broken by the supersymmetry breaking soft terms [15].

In the string derived models, the terms $\mu H_{d} H_{u}$, $\mu^{\prime} S^{2} / 2$ !, and $\kappa S^{3} / 3$ ! in superpotential in Eq. (3), which are forbidden at stringy tree level, might be generated due to the instanton effects. And the effective $\mu$ is about $M_{\text {string }} e^{-A}$ where $M_{\text {string }}$ is the string scale around $10^{17} \mathrm{GeV}$. So, the $\mu$ problem in the MSSM is solved if $A \sim 33$ [9]. Similar result holds for $\mu^{\prime}$. However, the $\kappa S^{3} / 3$ ! term generated from instanton effects might be highly suppressed.

To construct the string inspired SEMSSM, we consider the models with an anomalous $U(1)_{A}$ gauge symmetry [14]. In string model building, there generically exists one anomalous $U(1)$ gauge symmetry in the heterotic string model building [14] or up to four in the
Type II orientifold model building [16]. The corresponding anomalies are cancelled by the (generalized) GreenSchwarz mechanism [17]. We introduce a SM singlet field $\phi$ with $U(1)_{A}$ charge -1 . To cancel the Fayet-Iliopoulos term of $U(1)_{A}$, we assume that $\phi$ obtains a VEV so that the $U(1)_{A}$ D-flatness and supersymmetry can be preserved. Interestingly, $\langle\phi\rangle / M_{\mathrm{Pl}}$ is about 0.22 , where $M_{\mathrm{Pl}}$ is the Planck scale [14]. Moreover, to break the supersymmetry, we introduce a hidden sector superfield $Z$ whose $\mathrm{F}$ component acquires a VEV around $10^{21} \mathrm{GeV}^{2}$.

We assume that the $U(1)_{A}$ charges for $S$ and $Z$ are $n+p / q$ and $m+p^{\prime} / q^{\prime}$, respectively, where $m$ and $n$ are integers, $(p, q)$ and $\left(p^{\prime}\right.$ and $\left.q^{\prime}\right)$ are relatively prime positive integers, or $p / q$ or $p^{\prime} / q^{\prime}$ is zero. To have the $h S H_{d} H_{u}$ term in superpotential, we also assume that the total $U(1)_{A}$ charges for $H_{d}$ and $H_{u}$ are $-n-p / q$, but we do not give the explicit charges for $H_{d}$ and $H_{u}$ which are irrelevant to our discussions. Moreover, if $m+p^{\prime} / q^{\prime}$ is non-zero, the gaugino masses can not be generated via F-terms $Z W^{\alpha} W_{\alpha} / M_{\mathrm{Pl}}$. To have the gaugino masses, we can introduce another $U(1)_{A}$-uncharged hidden-sector superfield $Z^{\prime}$ whose $\mathrm{F}$ component acquires a VEV. In fact, in the string model building, both dilaton and moduli fields can break the supersymmetry due to their F-component VEVs. In addition, the supersymmetry breaking soft mass terms in $V_{\text {soft }}^{I}$ can be generated via D-term operators

$$
\int d^{4} x d^{2} \theta d^{2} \bar{\theta} \frac{\bar{Z} Z}{M_{\mathrm{Pl}}^{2}}\left(|S|^{2}+\left|H_{d}\right|^{2}+\left|H_{u}\right|^{2}\right),
$$

where for simplicity we neglect the coefficients of these operators in such kind of discussions in this paper. The first term $A_{h} h S H_{d} H_{u}$ in $V_{\text {soft }}^{I I}$ can be generated via the following F-term operator

$$
\int d^{4} x d^{2} \theta \frac{Z\left(\text { or } Z^{\prime}\right)}{M_{\mathrm{Pl}}} h S H_{d} H_{u}+\text { H.C. . }
$$

Model $A$ - We choose the following $U(1)_{A}$ charges for $S$ and $Z$

$$
m+n=47, \quad p / q=1 / 5, \quad p^{\prime} / q^{\prime}=4 / 5 .
$$

Then the $U(1)_{A}$ allowed renormalizable superpotential is

$$
W=h S H_{d} H_{u} .
$$

The additional supersymmetry breaking soft term $V_{\text {soft }}^{I I}$ can be generated via the following operator (the other operators are forbidden by $U(1)_{A}$ or negligible)

$$
\int d^{4} x d^{2} \theta M_{\text {string }} Z S\left(\frac{\phi}{M_{\mathrm{Pl}}}\right)^{48}+\text { H.C. . }
$$

So, we have

$$
V_{s o f t}^{I I}=-\left(A_{h} h S H_{d} H_{u}+A_{X} m_{X}^{2} S+\text { H.C. }\right),
$$


where $A_{h} \sim A_{X} \sim 10^{2} \mathrm{GeV}$, and $m_{X}^{2} \sim 10^{4-6} \mathrm{GeV}^{2}$. Interestingly, the global $U(1)$ symmetry in the Higgs potential is indeed broken by the supersymmetry breaking soft term $A_{X} m_{X}^{2} S$.

Model $B$ - We choose the following $U(1)_{A}$ charges for $S$ and $Z$

$$
n=-22, \quad p / q=0, \quad m=0, \quad p^{\prime} / q^{\prime}=0 .
$$

The additional relevant F-term and D-term operators are

$$
\begin{gathered}
\int d^{4} x d^{2} \theta\left(M_{\text {string }} H_{d} H_{u}+Z H_{d} H_{u}\right)\left(\frac{\phi}{M_{\mathrm{Pl}}}\right)^{22} \\
+\int d^{4} x d^{2} \theta d^{2} \bar{\theta}\left(\bar{Z} S+\frac{\bar{Z} Z S}{M_{\mathrm{Pl}}}\right)\left(\frac{\bar{\phi}}{M_{\mathrm{Pl}}}\right)^{22}+\text { H.C. }
\end{gathered}
$$

Thus, the superpotential in Model B is

$$
W=h S H_{d} H_{u}+\mu H_{d} H_{u}+m^{2} S,
$$

where $\mu \sim 10^{2-3} \mathrm{GeV}$ and $m^{2} \sim 10^{4-6} \mathrm{GeV}^{2}$. And the supersymmetry breaking soft terms in $V_{\text {soft }}^{I I}$ are

$$
\begin{aligned}
V_{\text {soft }}^{I I}= & -\left(A_{h} h S H_{d} H_{u}+B \mu_{B} H_{d} H_{u}\right. \\
& \left.+A_{X} m_{X}^{2} S+\text { H.C. }\right),
\end{aligned}
$$

where $A_{h} \sim B \sim \mu_{B} \sim A_{X} \sim 10^{2-3} \mathrm{GeV}$, and $m_{X}^{2} \sim$ $10^{4-6} \mathrm{GeV}^{2}$.

Model $C$ - In Model B, we consider the gauge mediated supersymmetry breaking scenario where the VEV of $\mathrm{F}$ component of $Z$ can be about $10^{10} \mathrm{GeV}^{2}$. And then the tadpole term $m^{2} S$ in the superpotential can be neglected. Thus, the superpotential in Model $\mathrm{C}$ is

$$
W=h S H_{d} H_{u}+\mu H_{d} H_{u}
$$

And the supersymmetry breaking soft terms in $V_{\text {soft }}^{I I}$ are

$$
V_{\text {soft }}^{I I}=-\left(A_{h} h S H_{d} H_{u}+B \mu_{B} H_{d} H_{u}\right) .
$$

Model $\mathrm{C}$ can also be considered as the string derived model with $h S H_{d} H_{u}$ superpotential term where the extra $\mu H_{d} H_{u}$ term arises from instanton effects [9].

Model $D$ - We choose the following $U(1)_{A}$ charges for $S$ and $Z$

$$
n=11, \quad p / q=1 / 2, \quad m=0, \quad p^{\prime} / q^{\prime}=0 .
$$

The additional relevant F-term operators are

$$
\int d^{4} x d^{2} \theta\left(M_{\text {string }} S^{2}+Z S^{2}\right)\left(\frac{\phi}{M_{\mathrm{Pl}}}\right)^{23}+\text { H.C. . }
$$

So, the superpotential is

$$
W=h S H_{d} H_{u}+\frac{\mu^{\prime}}{2 !} S^{2},
$$

TABLE I: The Higgs VEVs, and the charged, CP-even, and $\mathrm{CP}$-odd Higgs boson masses in $\mathrm{GeV}$ at tree level.

\begin{tabular}{|c|c|c|c|c|c|c|c|c|c|}
\hline Model & $\left\langle H_{d}^{0}\right\rangle$ & $\left\langle H_{u}^{0}\right\rangle$ & $\langle S\rangle$ & $H^{ \pm}$ & $H_{1}^{0}$ & $H_{2}^{0}$ & $H_{3}^{0}$ & $A_{1}^{0}$ & $A_{2}^{0}$ \\
\hline A & 119 & 127 & 213 & 205 & 67 & 196 & 210 & 127 & 251 \\
\hline B & 123 & 123 & 188 & 179 & 45 & 184 & 206 & 142 & 214 \\
\hline C & 123 & 123 & 161 & 165 & 66 & 148 & 171 & 31 & 214 \\
\hline D & 120 & 126 & 167 & 176 & 67 & 145 & 181 & 39 & 225 \\
\hline
\end{tabular}

where $\mu^{\prime} \sim 10^{2} \mathrm{GeV}$. And the supersymmetry breaking soft terms in $V_{\text {soft }}^{I I}$ are

$$
V_{\text {soft }}^{I I}=-\left(A_{h} h S H_{d} H_{u}+\frac{1}{2 !} B^{\prime} \mu_{B}^{\prime} S^{2}+\text { H.C. }\right) \text {, }
$$

where $A_{h} \sim B^{\prime} \sim \mu_{B}^{\prime} \sim 10^{2-3} \mathrm{GeV}$.

Model $\mathrm{D}$ can be considered as the string derived model with $h S H_{d} H_{u}$ superpotential term where the extra $\mu^{\prime} S^{2} / 2$ ! term arises from instanton effects [9]. However, there exists a $Z_{4}$ symmetry in Model D, where $H_{d}$ and $H_{u}$ have charge 1 , and $S$ has charge 2 . To avoid the domain wall problem after symmetry breaking, we can turn on tiny instanton effects to break the $Z_{4}$ symmetry by generating small high-dimensional operators, and then we can dissolve the domain wall.

Phenomenological Consequences - We shall calculate the Higgs boson masses, the chargino and neutralino masses at tree level in our models where we neglect the loop corrections for simplicity. The input parameters with dimensions of mass or mass-squared are chosen in arbitrary units. After finding an acceptable minimum they are rescaled so that $\sqrt{\left\langle H_{d}^{0}\right\rangle^{2}+\left\langle H_{u}^{0}\right\rangle^{2}} \simeq$ $174.1 \mathrm{GeV}$. For Model A, we choose: $h=0.7, m_{H_{d}}^{2}=$ $-0.1, m_{H_{u}}^{2}=-0.2, m_{S}^{2}=0.1, A_{h}=1.0, A_{X}=0.68$, $m_{X}^{2}=0.6$. And the VEVs for the Higgs fields at the minimum are $\left\langle H_{d}^{0}\right\rangle=0.7031,\left\langle H_{u}^{0}\right\rangle=0.75$, and $\langle S\rangle=1.2563$. For Model B, we choose: $h=0.7$, $\mu=-0.2, m^{2}=-0.3, m_{H_{d}}^{2}=-0.1, m_{H_{u}}^{2}=-0.1$, $m_{S}^{2}=0.1, A_{h}=0.6, B=-0.1, \mu_{B}=-0.2, A_{X}=-1.9$, $m_{X}^{2}=-0.3$. And the VEVs for the Higgs fields are $\left\langle H_{d}^{0}\right\rangle=0.8625,\left\langle H_{u}^{0}\right\rangle=0.8625$, and $\langle S\rangle=1.3156$. For Model C, we choose: $h=0.7, \mu=-0.1, m_{H_{d}}^{2}=-0.1$, $m_{H_{u}}^{2}=-0.1, m_{S}^{2}=-0.6, A_{h}=2.0, B=-0.6$, $\mu_{B}=-0.1$. And the VEVs for the Higgs fields are $\left\langle H_{d}^{0}\right\rangle=1.5875,\left\langle H_{u}^{0}\right\rangle=1.5875$, and $\langle S\rangle=2.075$. For Model D, we choose: $h=0.7, \mu^{\prime}=-0.3, m_{H_{d}}^{2}=-0.1$, $m_{H_{u}}^{2}=-0.4, m_{S}^{2}=-0.68, A_{h}=2.0, B^{\prime}=-0.6$, $\mu_{B}^{\prime}=-0.3$. And the VEVs for the Higgs fields are $\left\langle H_{d}^{0}\right\rangle=1.6375,\left\langle H_{u}^{0}\right\rangle=1.7203$, and $\langle S\rangle=2.275$.

We present the Higgs VEVs, the charged Higgs boson $\left(H^{ \pm}\right)$mass, CP-even Higgs boson $\left(H_{1}^{0}, H_{2}^{0}\right.$, and $\left.H_{3}^{0}\right)$ masses, and CP-odd Higgs boson $\left(A_{1}^{0}\right.$ and $\left.A_{2}^{0}\right)$ masses 
TABLE II: The chargino and neutralino masses in GeV.

\begin{tabular}{|c|c|c|c|c|c|c|c|c|}
\hline Model & $M_{i}$ & $\tilde{\chi}_{1}^{ \pm}$ & $\tilde{\chi}_{2}^{ \pm}$ & $\tilde{\chi}_{1}^{0}$ & $\tilde{\chi}_{2}^{0}$ & $\tilde{\chi}_{3}^{0}$ & $\tilde{\chi}_{4}^{0}$ & $\tilde{\chi}_{5}^{0}$ \\
\hline $\mathrm{A}$ & $>0$ & 115 & 334 & 68 & 88 & 175 & 217 & 336 \\
\hline $\mathrm{A}$ & $<0$ & 163 & 314 & 68 & 156 & 169 & 217 & 314 \\
\hline $\mathrm{B}$ & $>0$ & 75 & 328 & 56 & 81 & 167 & 184 & 330 \\
\hline $\mathrm{B}$ & $<0$ & 118 & 315 & 81 & 125 & 156 & 184 & 316 \\
\hline $\mathrm{C}$ & $>0$ & 76 & 329 & 58 & 80 & 167 & 185 & 330 \\
\hline $\mathrm{C}$ & $<0$ & 120 & 315 & 80 & 127 & 156 & 185 & 316 \\
\hline $\mathrm{D}$ & $>0$ & 87 & 330 & 60 & 68 & 169 & 200 & 331 \\
\hline $\mathrm{D}$ & $<0$ & 132 & 315 & 61 & 138 & 156 & 200 & 315 \\
\hline
\end{tabular}

in Table I. Interestingly, the couplings of the CP-even Higgs boson $H_{1}^{0}$ and $H_{2}^{0}$ with $Z^{0}$ gauge boson almost vanish, and only the heaviest CP-even Higgs boson $H_{3}^{0}$ can couple to $Z^{0}$ [15]. Thus, the fine-tuning problem for the lightest CP-even Higgs boson mass in the MSSM from the LEP constraints can be completely relaxed, and we have new Higgs physics at the LHC and ILC because $H_{3}^{0}$ has mass around $190 \mathrm{GeV}$. Moreover, to calculate the chargino and neutralino masses, we choose the positive and negative gaugino masses $M_{1}$ and $M_{2}$ for $U(1)_{Y}$ and $S U(2)_{L}:$ (1) $M_{1}=150 \mathrm{GeV}$, and $M_{2}=300 \mathrm{GeV}$; (2) $M_{1}=-150 \mathrm{GeV}$, and $M_{2}=-300 \mathrm{GeV}$. The masses for charginos $\left(\tilde{\chi}_{1}^{ \pm}\right.$and $\left.\tilde{\chi}_{2}^{ \pm}\right)$and neutralinos $\left(\tilde{\chi}_{i}^{0}\right.$ with $i=1,2, \ldots, 5)$ are given in Table II.

Conclusions - In the string derived SEMSSM, $h S H_{d} H_{u}$ is the only allowed superpotential term at stringy tree level. Then there exist an additional global $U(1)$ symmetry in the Higgs potential, and the axion problem. We propose the string inspired SEMSSM in which the global $U(1)$ symmetry is broken by the additional superpotential terms or supersymmetry breaking soft terms. The extra superpotential terms can be obtained via instanton effects in the string derived models. With anomalous $U(1)_{A}$ gauge symmetry, we present four simple and concrete SEMSSM. In these models, we can naturally solve the $\mu$ problem and the fine-tuning problem for the lightest CP-even Higgs boson mass in the MSSM, and generated the observed baryon asymmetry via electroweak baryogenesis. In addition, we calculate the Higgs boson masses, chargino masses and neutralino masses at tree level, and predict the new Higgs physics which can be tested at the LHC and ILC. Confirmation one of these models at the future colliders might give us indirect implication of string theory.

Acknowledgments - This research was supported in part by the Cambridge-Mitchell Collaboration in Theoretical Cosmology.

[1] S. Dimopoulos and H. Georgi, Nucl. Phys. B 193, 150 (1981); S. Dimopoulos, S. Raby and F. Wilczek, Phys. Rev. D 24, 1681 (1981); N. Sakai, Z. Phys. C 11, 153 (1981); L. E. Ibanez and G. G. Ross, Phys. Lett. B 105, 439 (1981); M. B. Einhorn and D. R. T. Jones, Nucl. Phys. B 196, 475 (1982); W. J. Marciano and G. Senjanovic, Phys. Rev. D 25, 3092 (1982).

[2] U. Amaldi, W. de Boer and H. Furstenau, Phys. Lett. B 260, 447 (1991); J. R. Ellis, S. Kelley and D. V. Nanopoulos, Phys. Lett. B 249, 441 (1990); P. Langacker and M. X. Luo, Phys. Rev. D 44, 817 (1991); C. Giunti, C. W. Kim and U. W. Lee, Mod. Phys. Lett. A 6 (1991) 1745.

[3] Y. Okada, M. Yamaguchi and T. Yanagida, Prog. Theor. Phys. 85, 1 (1991); J. R. Ellis, G. Ridolfi and F. Zwirner, Phys. Lett. B 257, 83 (1991); H. E. Haber and R. Hempfling, Phys. Rev. Lett. 66, 1815 (1991); M. Carena, J. R. Espinosa, M. Quiros and C. E. M. Wagner, Phys. Lett. B 355, 209 (1995).

[4] P. Fayet, Nucl. Phys. B 90, 104 (1975); H. P. Nilles, M. Srednicki and D. Wyler, Phys. Lett. B 120, 346 (1983); J. M. Frere, D. R. T. Jones and S. Raby, Nucl. Phys. B 222, 11 (1983); J. P. Derendinger and C. A. Savoy, Nucl. Phys. B 237, 307 (1984).

[5] R. Dermisek and J. F. Gunion, Phys. Rev. Lett. 95, 041801 (2005).

[6] M. Pietroni, Nucl. Phys. B 402 (1993) 27.

[7] C. Panagiotakopoulos and K. Tamvakis, Phys. Lett. B 469, 145 (1999); C. Panagiotakopoulos and A. Pilaftsis, Phys. Rev. D 63, 055003 (2001); A. Dedes, C. Hugonie, S. Moretti and K. Tamvakis, Phys. Rev. D 63, 055009 (2001).

[8] M. Cvetic, D. A. Demir, J. R. Espinosa, L. L. Everett and P. Langacker, Phys. Rev. D 56, 2861 (1997) [Erratumibid. D 58, 119905 (1998)]; J. Erler, P. Langacker and T. Li, Phys. Rev. D 66, 015002 (2002); V. Barger, P. Langacker, H. S. Lee and G. Shaughnessy, Phys. Rev. D 73, 115010 (2006).

[9] R. Blumenhagen, M. Cvetic and T. Weigand, Nucl. Phys. B 771, 113 (2007); L. E. Ibanez and A. M. Uranga, JHEP 0703, 052 (2007).

[10] V. Braun, Y. H. He, B. A. Ovrut and T. Pantev, Phys. Lett. B 618, 252 (2005); V. Bouchard and R. Donagi, Phys. Lett. B 633, 783 (2006); V. Bouchard, M. Cvetic and R. Donagi, Nucl. Phys. B 745, 62 (2006).

[11] C. M. Chen, T. Li and D. V. Nanopoulos, Nucl. Phys. B 751, 260 (2006).

[12] E. Witten, Phys. Lett. B 155, 151 (1985); T. Li, J. L. Lopez and D. V. Nanopoulos, Phys. Rev. D 56, 2602 (1997).

[13] D. Cremades, L. E. Ibanez and F. Marchesano, JHEP 0307, 038 (2003); M. Cvetic and I. Papadimitriou, Phys. Rev. D 68, 046001 (2003) [Erratum-ibid. D 70, 029903 (2004)]; S. A. Abel and A. W. Owen, Nucl. Phys. B 663, 197 (2003).

[14] H. K. Dreiner, H. Murayama and M. Thormeier, Nucl. Phys. B 729, 278 (2005), and references therein.

[15] T. Li, in preparation.

[16] L. E. Ibanez, F. Marchesano and R. Rabadan, JHEP 0111, 002 (2001).

[17] M. B. Green and J. H. Schwarz, Phys. Lett. B 149, 117 
(1984). 